UCRL --101486

DE91 004192

\title{
INTEGRATED OPTICAL DEVICES FOR PHOTONICS INSTRUMENTATION SYSTEMS
}

\author{
G. M. MCWRIGHF \\ D. A. LOFAW \\ M. LOWRY \\ W. TINDALL
}

\section{This paper is being prepared for submittal \\ to the International Congress on Optical \\ Science and Engineering, \\ The Hague, Netherlands}

March 12 thru 16,1990

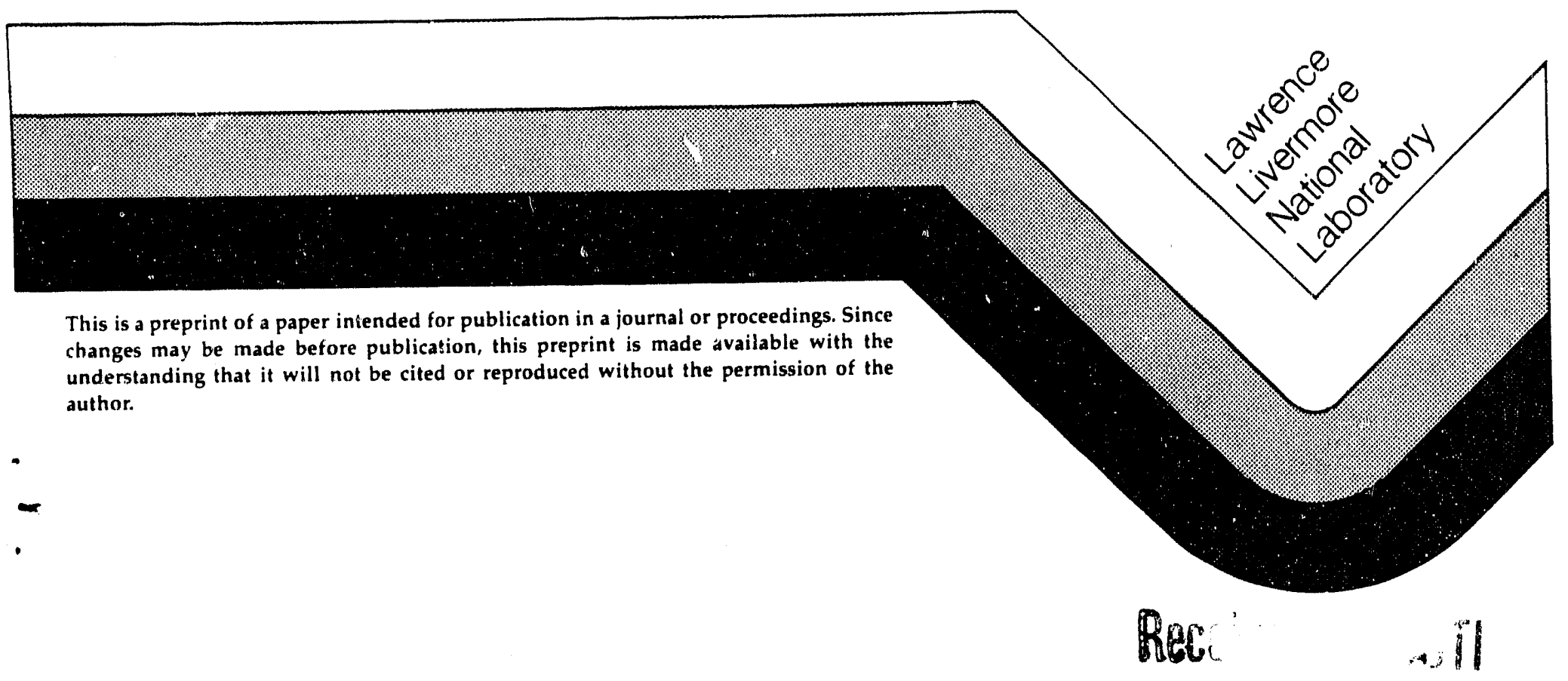




\section{DISCI.AIMF.R}

This document was prepured as an uccount of work sponsored hy an agency of the Inited Stalesi Government. Neither the Liniled States Government nor the University of California nor ans of their employees, makes any narranty. express or implied. or assumes any legul liabilite or responsibility for the accuracs, completeness, or usefulness of any information. apparatus. product, or process disclosed. or represents that its use would not infringe privately anned rights. Reference herein to any specific commercial products, process, or service by trade name. Irademark. manufacturer, or otherwise. does not necessaril! constitute or impls its endorsement. recommendation. or favoring by the I nited Sitales (jovernment or the Iniversity of California. The viens and upinions of authors espressed herein do not necessarily state or reflect those of the I'nited States (jovernment or the I niversity of California. and shall not be used for advertising or protuct endorsement purpenses. 
Integrated optical devices for photonics instrumentation systems

\author{
G. McWright, D. Lafaw, M. Lowry, and W. Tindall \\ Lawrence Livermore National Laboratory, \\ P.O. Box 808, Mail Stop L-156, Livermore, CA 94550
}

\begin{abstract}
We discuss the design, fabrication, and evaluation of high speed integrated optical devices for application to photonics instrumentation systems. Specifically, we have demonstrated integrated optical devices with bandwidths in excess of $25 \mathrm{GHz}$ and implemented these devices in single-shot, streak camera based recording schemes.

\section{LNTRRODUCTRON}

Integrated optical devices have reached a high level of technological maturity and are rapidly finding application in a number of different optical systems (1). While fiber-optic based telecommunication systems have been the driving force behind much of the early work done in integrated optical device research, the devices are also finding application in many other types of systems. Our interest in integrated optics stems from a need to develop very high speed data acquisition systems; indeed, integrated optics is the preferred technology for transmitting high density, high speed, and high fidelity information (2). Here, we describe the design, fabrication, and evaluation of high speed integrated optical devices for application to single-shot, photonics instrumentation systems.
\end{abstract}

Specifically, we have been developing high speed integrated optical diagnostic systems which can be used to transmit an analog signal from a remote radiation sensor (Fig. 1). Many conventional diagnostic systems use electrical cables. Electrical cable is lossy and dispersive, so our electrical cable based systems, which frequently use electrical cables of hundreds of meters in length, are generally not satisfactory for transmitting temporal information below $1 \mathrm{~ns}$. Furthermore, electrical cables are very susceptible to interference from ionizing radiation, which is present in many of: the envircnments where our diagnostic signals are present.

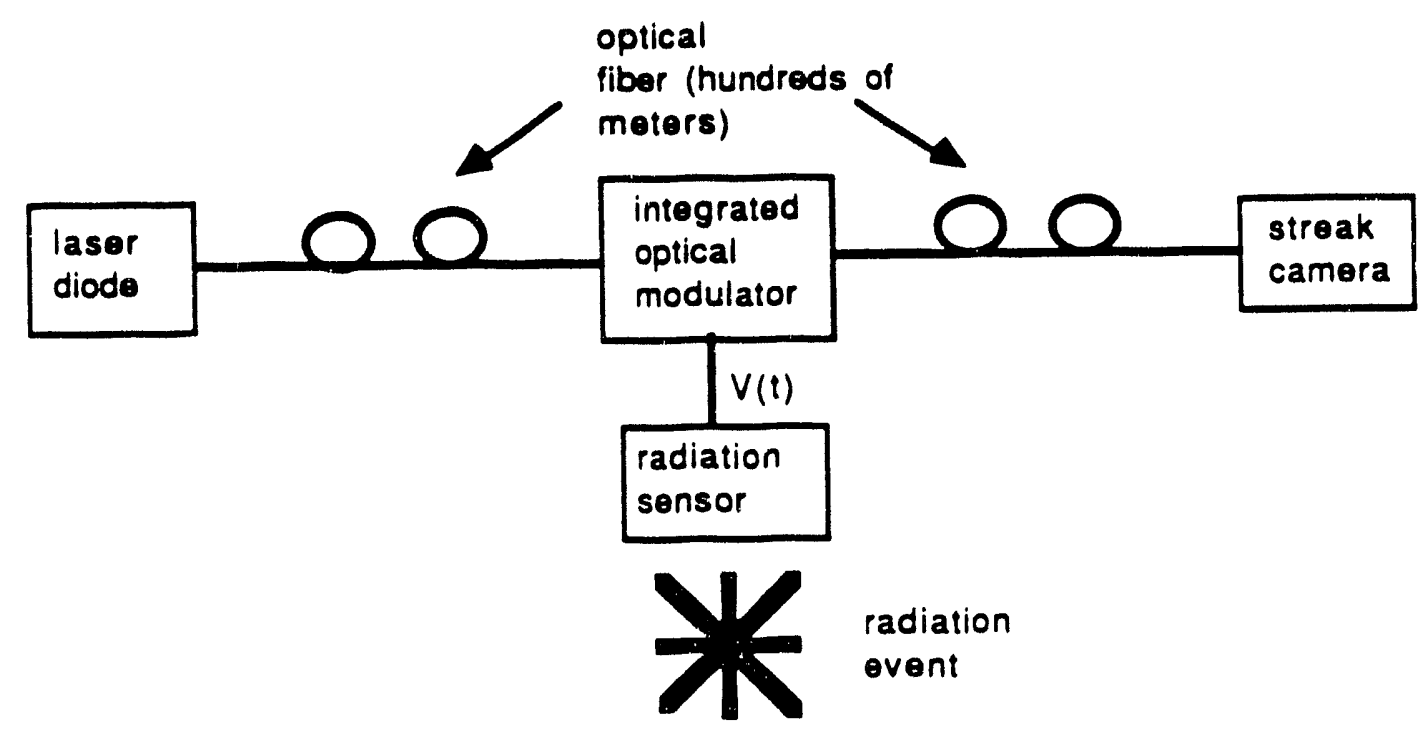

Fig. 1. High-spied integrated optical diagnostic system.

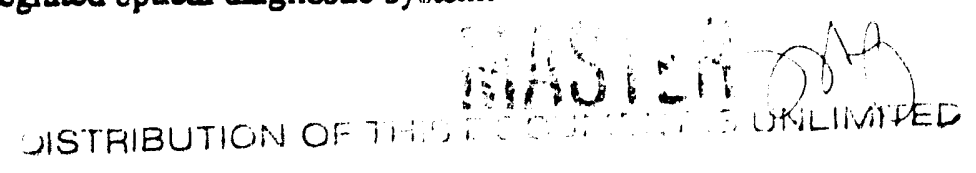


Both of these problems with electrical-cable based transmission systems can be circumvented with an integrated optical based diagnostic system. As shown in Fig. 1, the integrated optical based sytem uses optical fibers, in addition to several other components: a laser diode, an integrated optical external modulator, a radiation sensor which serves as an electrical driver for the modulator, and a streak camera recording system. We have focused specifically on photonics instrumentation systems which use streak camera recording systems, because streak cameras are capable of high-speed ( 20 ps), single-shot operation. However, the photocathode in the streak camera is not sensitive in the popular 1.3- $\mu \mathrm{m}$ region of the electromagnetic spectrum; thus, this streak camera based system operates at a wavelength of 0.8- $\mu \mathrm{m}$. The focus of this paper is the integrated optical modulator. Specifically, we describe device design, fabrication, and evaluation, and $a^{2}$, discuss implementation of these devices in photonics instrumentation systems.

\section{DEVICEEABRICATTON}

For our integrated optical diagnostic system, we chose to focus on one particular type of integrated optical modulator, the Mach-Zehnder, because this design offers high bandwidth, environmental robustness, and ease of fabrication. The Mach-Zehnder is an electro-optically tunable interferometer. It is formed by photolithographic techniques similar to those used in semiconductor chip manufacture. First, optical waveguides are defined in the lithium niobate electrooptic crystal by the in-diffusion of titanium, then gold electrodes are deposited on the waveguides. The structure is designed so that the light splits at the input $Y$-branch into two beams that propagate independently for a few millimeters and then recombine at the output $Y$-branch. If no electric field is applied, the beams arrive at the output $Y$-branch in phase and recombine without loss. However, application of an electric field introduces a pach length difference between the two beams so that the combined beam does not propagate efficienuly. $X$-cut, y-propagating lithium niobate is used for our Mach-Zehnder fabrication. In order to achieve high bandwidths, traveling wave electrodes are used, and the crystal thickness is $375 \mu \mathrm{m}$. The titanium width before diffusion is $3.3 \mu \mathrm{m}$, and the thickess is $440 \AA$. Diffusion is performed in a wet oxygen environment at $980 \mathrm{C}$ for 6.5 hours.

Subsequently, a $2000 \AA$ silicon dirxide layer is deposited on the lithium niobate in order to reduce the optical insertion loss of the structure, the lithium niobate end-faces are optically polished, and gold electrodes are electroplated to a thickness of approximately $4 \mu \mathrm{m}$. The electrode width is $19 \mu \mathrm{m}$, and the gap is $6 \mu \mathrm{m}$, thus yielding a characteristic impedance of approximately $23 \mathrm{ohms}$. The electrode length is $3 \mathrm{~mm}$.

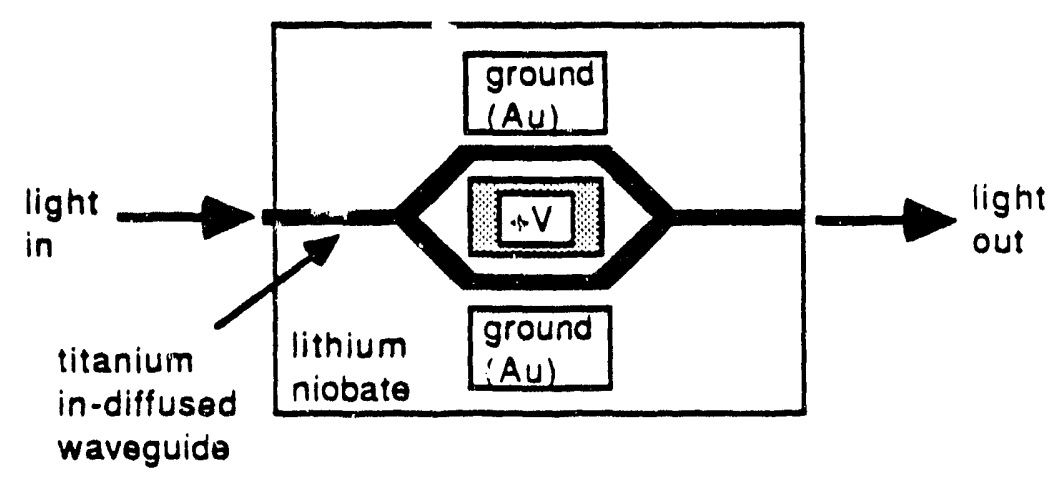

Fig. 2. Schematic representation of Mach-Zehnder modulator.

Polarization preserving optical fibers are then atached using UV curing optical epoxy, and the device is packaged. As shown in Fig. 3, the optical fibers are strain-relieved in lithium niobate blocks which are crystallographically matched to the orientation of the lithium niobate substrate ( $x$-cul, $y$-propagating). This design ensures an environmentally robust, thermally stable package. Typical insertion losses are in the range $3-5 \mathrm{~dB}$. Furchermore, preliminary temperature cycling studies indicate that the optical insertion loss varies by less than ! $\mathrm{AB}$ over the temperature range -40 to $+80 \mathrm{C}$. The electrical input is a Wiltron $\mathrm{K}$-connector, which is soldered to the gold electrode using indium solder. In addition, the traveling wave electrode is terminated in its characteristic impedance ( $23 \mathrm{ohms}$ ) with a high 
quality, microwave chip resistor. This cornection is also made with indium solder to insure good microwave characteristics. Electrooptic measurements are described in detail in the next section.

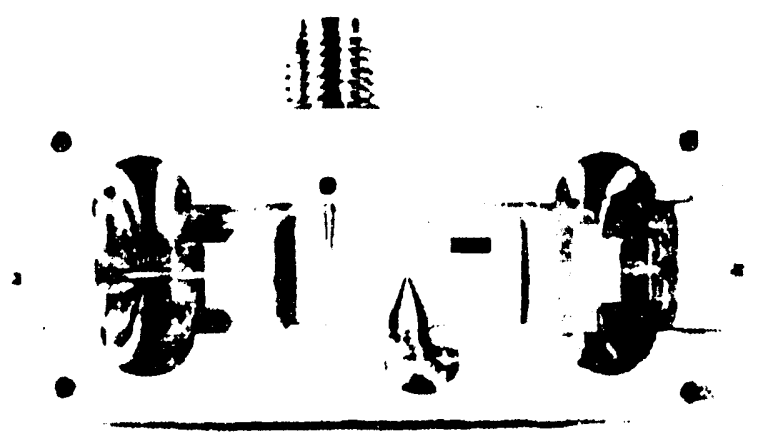

Fig. 3. Picture of packaged Mach-Zehnder.

\section{ELECTROOPTIC EVALUATION}

The de switching characteristics of the Mach-Zehnder are measured by over-Ariving the Mach-Zehnder with a triangular wave voltage pulse and monitoring the resulting intensity modulated light signal on a photodetector (3). If the triangular wave voltage pulse has sufficient amplitude, the modulator is driven through several oscillatory cycles. Consequently, the de switching voltage can be determined. Fig. 4 shows a typical de switching voltage measurement; in this case, a 20 volt triangular wave voltage pulse is applied to the modulator and the resulting modulated optical output (the noise on the modulated optical ouput is the result of vibrations in our optical system). Thus, the dc switching voltage is 10 volts.

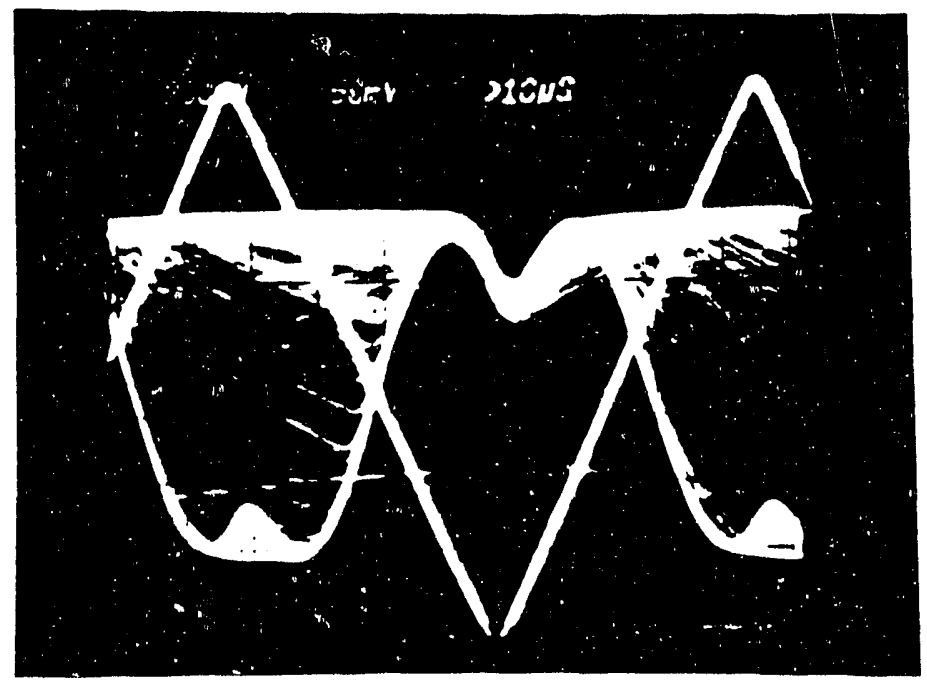

Fig. 4. DC switching voltage measurement for Mach-Zehnder modulator.

The frequency response of the modulator was measured using the so-calied "swept-frequency" technique (4). This technique is in general use in the optoelectronic community, and yields an accurate representation of the electrooptical characteristics of the device without the need for a high frequeicy detection system. In this technique a high frequency modulating signal $(0.01-26 \mathrm{GHz})$ is square wave modulated by a low frequency $(5 \mathrm{kHz})$ signal. The amplitude 
modulated microwave signal is then applied to the modulator, and the envelope of the modulated optical output is detected with a low speed photodetector and lock-in amplifier. The variation in the amplitude of the envelope with frequency yields the frequency response of the modulator. Frequency response measurements for a typical device are shown in Fig. 5 and indicate a useful frequency response in excess of $25 \mathrm{GHz}$.

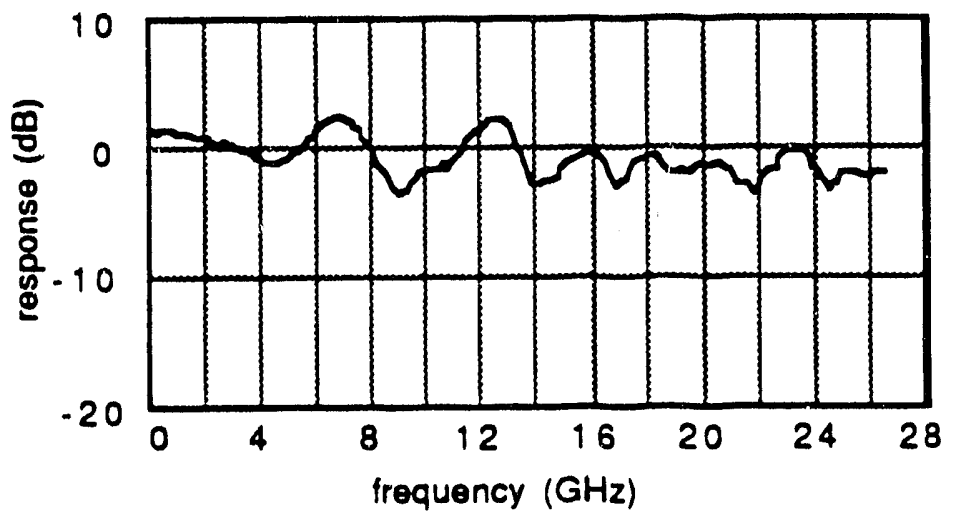

Fig. 5. Frequency response of the modulator.

\section{SYSTEM CHARACTERIZATION}

Next, we used a streak camera recording system to characterize the response of the modulator in the time domain. The experimental setup is similar to the arrangement shown in Fig. 1, except that the modulator was driven with a single, analog voltage pulse from a voltage pulser, rather than a radiation detector. The light source was a Sharp laser diode which operates at a wavelength of $830 \mathrm{~nm}$. The laser is pigtailed with single mode, polarization preserving fiber, which has been slant polished to minimize back-reflections into the laser cavity. To circumvent photorefractive damage in the lithium niobate crystal, the laser was pulsed on with approximately 5 millisecond long electrical pulses, thus yielding a peak power out of the pigtail of approximately $17.6 \mathrm{mw}$. The modulator was DC-biased to its linear operating region with -6.5 volts, and driven with a 4.2 -volt, Gaussian shaped electrical pulse (FW:-IM 75 psec) from a Picosecond Laboratories Pulser. Our streak camera is a Thomson model TSN 506 with a Photometrics CCD readout and a time response of approximely 20 ps. The CCD chip has $388 \times 576$ pixels, is thermoelectrically cooled, and has a 14-bit analog-to-digital conversion. The streak camera had two fiber inputs: one channel from the modulator, and a second channel from a light pulse train (a comb generator) for timing. For our measurements, a weighted 7-point column average of the optical streak was performed in the direction perpendicular to the time axis.

A streak camera trace of the modulated optical carrier is shown in Fig. 6. For reference, an oscilloscope trace of the voltage drive pulse is superimposed on the same plot. As indicated in Fig. 6, the modulated optical carrier has a FWHM of approximately 75 ps; thus, there appears to be litule temporal broadening of the modulated optical carrier. In addition, the modulated optical carrier faithfully reproduces the electrical drive signal, confirming that the modulator is operating in its linear region. We note that it is generally not accurate to use deconvolution by sum-of-the-squares to extract the impulse response of the modulator when it is driven by finite width pulses (5). However, these temporal results do tend to confirm the excellent high frequency characteristics of the modulator as measured with the swept-frequency lechnique. 


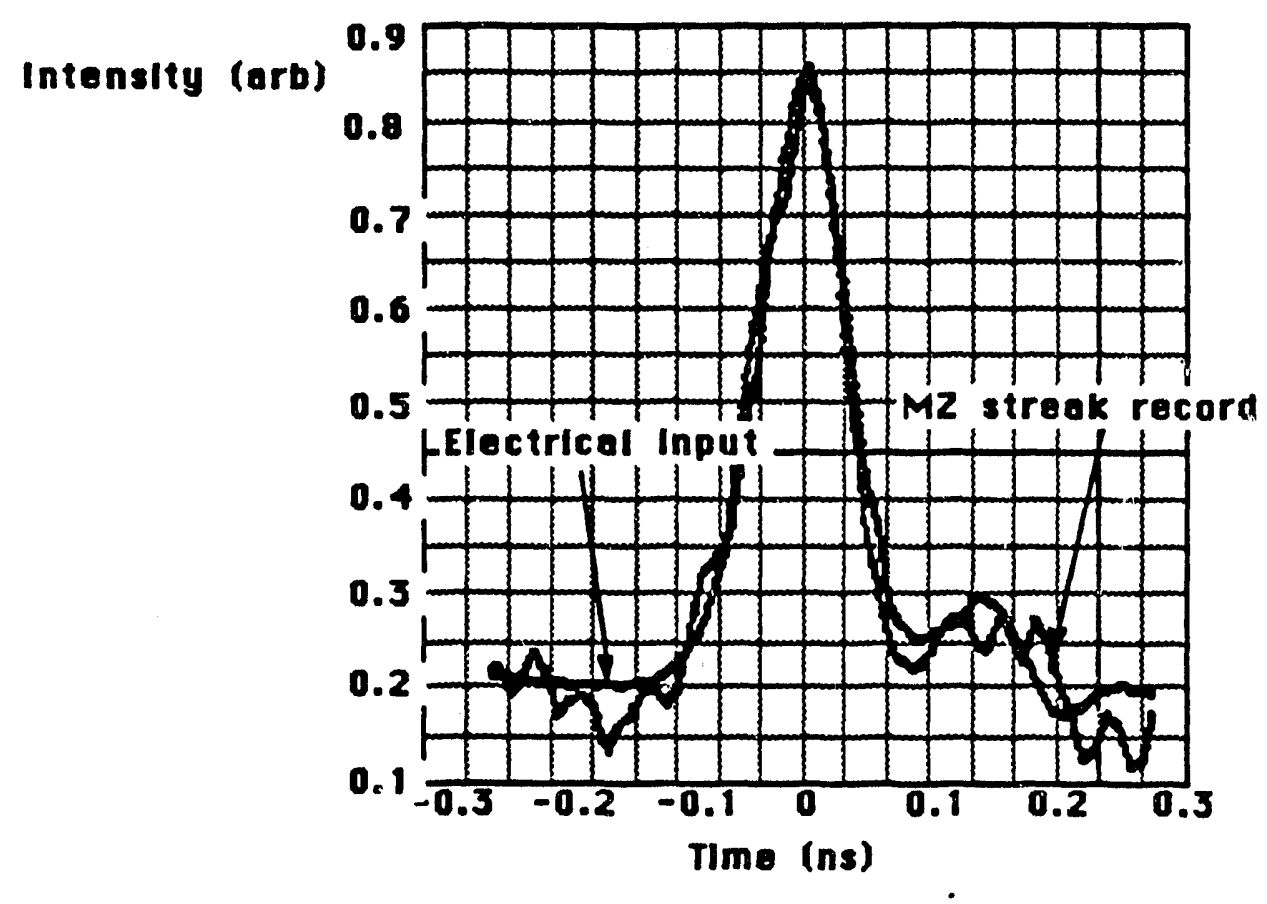

Fig. 6. Streak camera trace of modulated optical carrier. An oscilloscope trace of the voltage drive pulse is shown for reference.

\section{CONCLUSIONS}

We have demonstrated an integrated optical based instrumentation system for the measurement of high speed, single analog voltage transient signals. The system uses a laser diode, a high speed Mach-Zehnder modulator, and a streak camera recording system. The modulator, itself, exhibits low switching voltage (< 10 volts), low optical insertion loss $(<3 \mathrm{~dB})$, and high bandwidth $(25 \mathrm{GHz})$. The complete system has a $<50$ ps time response, good fidelity, and large dynamic range, and should continue to play a useful role in the collection and analysis of high speed transient signals.

\section{ACKNOWLEDGMENTS}

The support and encouragement of J. Balch and M. Pocha is gratefully acknowledged. This work was performed under the auspices of the U.S. Department of Energy by the Lawrence Livermore National Laboratory under Contract No. W-7405-Eng-48.

\section{I.REFERENCES}

1. R. Alferness, "Waveguide Electrooptic Modulators," IEEE Transactions on Microwave Theory and Techniques, vol. MTT-30, pp. 1121-1137, August, 1982.

2. M. Lowry, G. Lancaster, R. Peterson, G. McWright, D. Nelson, and B. Kidd, "Precision Transfer Function Measurements of Integrated Optical Modulators," Optoelectronic Materials, Devices, Packaging, and Inserconnects II, G. McWright, Ed., Proceedings SPIE 994, pp. 205-211, 1988.

3. R. A. Becker, "Traveling-wave electro-optic modulator with maximum bandwidth-length product," Applied Physics Letters, vol. 45, pp. 1168-1170, 1984. 
4. S. Uehara, "Calibration of optical modulator frequency response with application to signal level control," Applied Optics, vol. 17, pp. 68-71, 1978.

5. S. K. Korotky and R. C. Alferness, "Time- and frequency-domain response of directional-coupler traveling-wave optical modulators," Journal of Lightwave Technology, vol LT-1, pp. 244-251, 1983. 

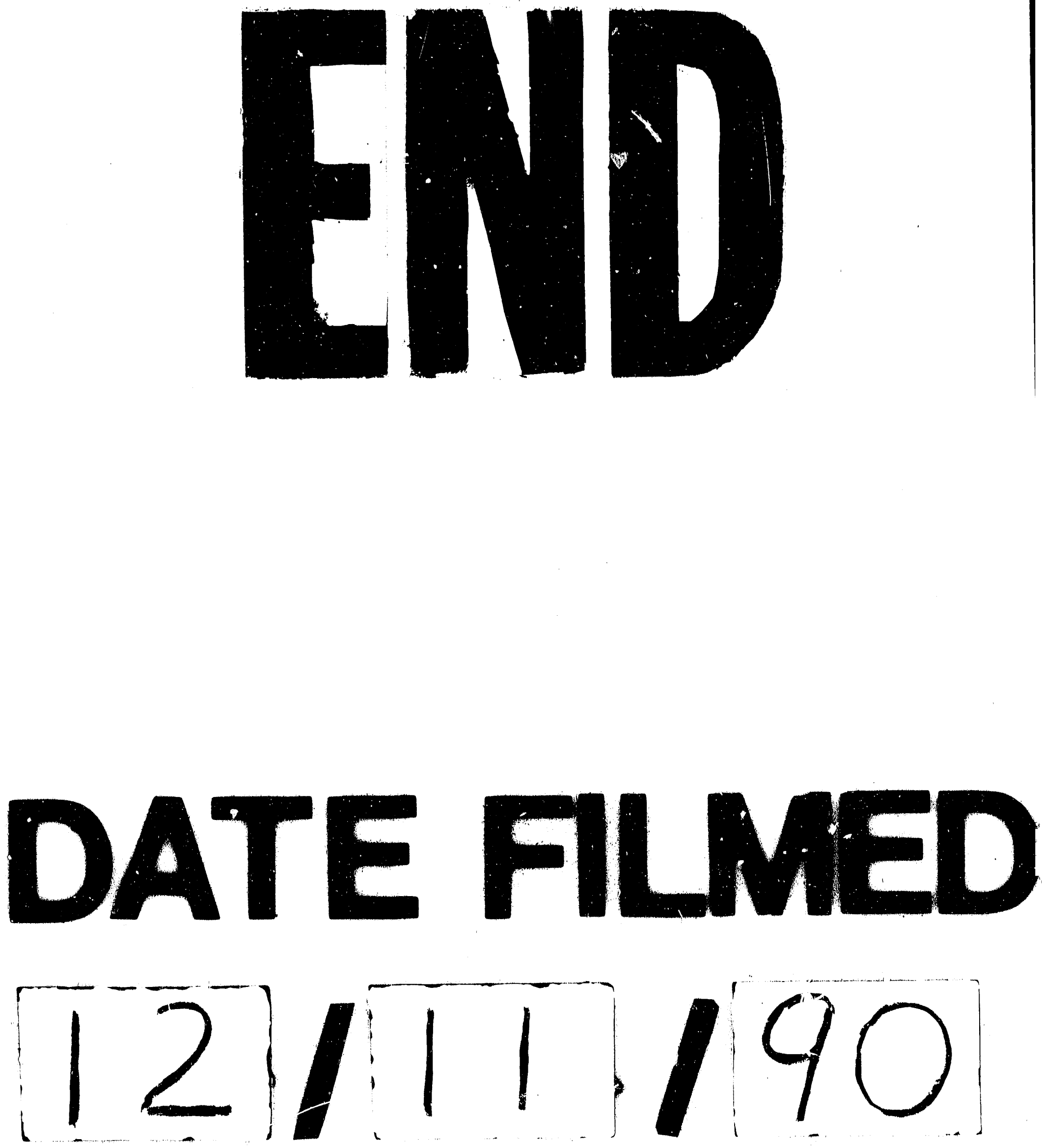
